\title{
Phase space Feynman path integrals
}

\author{
S. Albeverio \\ Institut für Angewandte Mathematik, Wegelerstrasse 6, 53115 Bonn, Germany \\ G. Guatteri, and S. Mazzucchi ${ }^{\mathrm{a})}$ \\ Dipartimento di Matematica, Università di Trento, 38050 Povo, Italia
}

(Received 21 December 2001; accepted for publication 8 February 2002)

A rigorous mathematical formulation of the "phase space Feynman path integral" is given in a general setting. This is then applied to yield a representation of solutions of the Schrödinger equation with potential depending both on the position and momentum variables. (C) 2002 American Institute of Physics.

[DOI: 10.1063/1.1470705]

\section{INTRODUCTION}

Let us consider the Schrödinger equation for the time evolution of a $d$-dimensional quantum particle:

$$
\left\{\begin{array}{l}
\dot{\psi}=-\frac{i}{\hbar} H \psi, \\
\psi(0, x)=\psi_{0}(x),
\end{array}\right.
$$

where $H=p^{2} / 2 m+V$ is the Hamiltonian of the system, $m>0$ is the mass of the particle, $\hbar$ is the (reduced) Planck' constant, and $p$ is the quantum mechanical momentum operator. Under suitable conditions on the potential $V$, in the position representation $H$ is realized as the self-adjoint operator in the Hilbert space $\mathcal{H}=\mathcal{L}_{2}\left(\mathbb{R}^{d}\right)$, obtained by closure of the operator

$$
\begin{gathered}
H \psi(x)=-\frac{\hbar^{2}}{2 m} \Delta \psi(x)+V(x) \psi(x), \quad \psi \in D(-\Delta) \cap D(V), \\
\psi \in \mathcal{H}, \quad \int_{\mathbb{R}^{d}}|\psi(x)|^{2} \mathrm{~d} x<+\infty,
\end{gathered}
$$

where $D(A)$ denotes the domain of the operator $A$ in $\mathcal{H}$ (see, e.g. Ref. 1). Let $G(t, x, y)$ be the Green function or propagator, namely the kernel of the unitary group $e^{-i t H / \hbar}$,

$$
\psi(t, x)=\int_{\mathbb{R}^{d}} G(t, x, y) \psi_{0}(y) \mathrm{d} y
$$

(see, e.g., Ref. 1 for a discussion on sufficient conditions for the existence of $G$ and for the properties of it). In 1942 Feynman gave a suggestive representation of the propagator and showed the connection between the classical Lagrangian description of the physical world and the quantum one. In fact the kernel of the unitary group can be heuristically computed by means of an infinite dimensional path integral of the following form:

$$
G(t, x, y)=\text { const } \int e^{(i / \hbar) S(\gamma)} \mathrm{d} \gamma,
$$

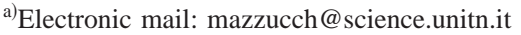


where the integration is on the set of paths starting in $y$ at time 0 and ending in $x$ at time $t$ and $S(\gamma)$ is the classical action of the system, evaluated on the path $\gamma$.

$$
S(\gamma)=\int_{0}^{t}\left(\dot{\gamma}(s)^{2} / 2 m-V(\gamma(s)) \mathrm{d} s .\right.
$$

Expression (2) lacks rigor, e.g., neither the constant in front of the integral nor the "infinite dimensional Lebesgue measure" $\mathrm{d} \gamma$ are well defined. Nevertheless a rigorous mathematical formulation of the Feynman functional can be given (see Refs. 2 and 3, and references therein; for other approches see also Refs. 4 and 5). Feynman himself (see Ref. 6) gave other heuristic representations of the integral, e.g., the heuristic integration over paths in phase space. For physical discussions of this concept see also Ref. 7.

The aim of the present paper is to give a mathematical definition of a "phase space path integral:" a Hamiltonian instead of a Lagrangian representation of the propagator. We want to give mathematical meaning to the Hamiltonian version of formula (2), namely to

$$
G(t, x, y)=\text { const } \int e^{(i / \hbar) S(q, p)} \mathrm{d} q \mathrm{~d} p,
$$

where $q(s), p(s), s \in[0, t]$ are paths in the phase space and $S$ is the action in the Hamiltonian fomulation: $S(q, p)=\int_{0}^{t}(\dot{q}(s) p(s)-H(q(s), p(s))) d s$.

The Hamiltonian formulation is more convenient for two reasons:

(1) for many classical systems it is better than the Lagrangian one;

(2) the discussion of the approach from quantum mechanics to classical mechanics, i.e., the study

of the behavior of physical quantities taking into account that $\hbar$ is small, is more natural in a Hamiltonian setting (see, e.g., Refs. 8 and 9 for a discussion of this behavior).

We note that an approach of phase space Feynman path integrals via analytic continuation of "phase space Wiener integrals" has been presented by Daubechies and Klauder. ${ }^{10}$ Analytic continuation was also used in other "path space" approaches, see Refs. 11 and 4, and references therein. Our approach is more direct in the spirit of Ref. 2.

\section{LIE-TROTTER PRODUCT FORMULA}

We first recall an abstract version of the Lie-Trotter product formula.

Lemma 1: Let $A$ and $B$ be self-adjoint operators in a Hilbert space $\mathcal{H}$ and let $A+B$ be essentially self-adjoint on $D(A) \cap D(B)$. Then

$$
s-\lim _{n \rightarrow \infty}\left(e^{i t a / n} e^{i t B / n}\right)^{n}=e^{i(A+B) t}, \quad t \in \mathbb{R} .
$$

Here $s-\lim$ is the strong operator limit. For a proof and a discussion of this lemma see e.g., Refs. 12 and 1.

Let $\mathcal{H}=L^{2}\left(\mathbb{R}^{d}\right)$ and let us consider a potential $V$ depending both on the position and on the momentum in the following way: $V=V_{1}(x)+V_{2}(p) . V_{1}$ is defined as a self-adjoint operator in $\mathcal{H}$, with its natural domain as a multiplication operator. $V_{2}$ is the operator in $\mathcal{H}$ with domain

$$
D\left(V_{2}(p)\right)=\left\{\psi \in \mathcal{H} \mid \alpha \rightarrow V_{2}(\alpha) \hat{\psi}(\alpha) \in \mathcal{H}\right\},
$$

where $\hat{\psi}$ is the Fourier transform of $\psi$. It coincides with the operator defined by functional calculus as $V_{2}(p)$, with $p$ the self-adjoint operator $-i \hbar \nabla$ in $\mathcal{H}$. $V$ is then the sum, as a self-adjoint operator in $\mathcal{H}$, of the self-adjoint operators $V_{1}$ and $V_{2}$. We assume that the functions $V_{1}$ and $V_{2}$ are such that the corresponding operators have a common dense domain of essentially self-adjointness $D$. This is the case, e.g., when $V_{1} \in L^{2}\left(\mathbb{R}^{d}\right)+L^{\infty}\left(\mathbb{R}^{d}\right), V_{2}$ is bounded measurable, and $D=C_{0}^{\infty}\left(\mathbb{R}^{d}\right)$ or $D=\mathcal{S}\left(\mathbb{R}^{d}\right)$. We assume, in order to apply lemma 1 , that $V_{1}, V_{2}$ are such that $-\left(\hbar^{2} / 2 m\right) \Delta+V_{2}$ and $-\left(\hbar^{2} / 2 m\right) \Delta+V_{1}+V_{2}$ are essentially self-adjoint on $D$. We denote by $H$ the closure of the 
latter operator. $H$ (which we also write simply as $\left.-\left(\hbar^{2} / 2 m\right) \Delta+V_{1}+V_{2}\right)$ is then the quantum Hamiltonian.

By lemma 1 we have then

$$
\begin{gathered}
\exp \left(-\frac{i t\left(p^{2} / 2 m+V\right.}{\hbar}\right)=s-\lim _{n \rightarrow \infty}\left(\exp \left(-\frac{i \epsilon\left(p^{2} / 2 m+V_{2}\right)}{\hbar}\right) \exp \left(-\frac{i \epsilon\left(V_{1}\right)}{\hbar}\right)\right)^{n}, \quad \epsilon \equiv \frac{t}{n}, \\
\psi(t)=\exp \left(-\frac{i t\left(p^{2} / 2 m+V\right.}{\hbar}\right) \psi_{0}=\lim _{n \rightarrow \infty}\left(\exp \left(-\frac{i \epsilon\left(p^{2} / 2 m+V_{2}\right)}{\hbar}\right) \exp \left(-\frac{i \epsilon V_{1}}{\hbar}\right)\right)^{n} \psi_{0}, \\
\psi_{0} \in \mathrm{C}_{0}^{\infty}\left(\mathbb{R}^{d}\right),
\end{gathered}
$$

(see, e.g., Refs. 12 and 13 for related uses of the Lie-Trotter formula).

By shifting from the position representation to the momentum representation and vice versa and assuming that $V_{1}$ and $V_{2}$ are continuous, we can write in the strong $L^{2}\left(\mathbb{R}^{d}\right)$-sense, for all $t$ $>0$ :

$$
\begin{aligned}
\psi(t, x)= & \lim _{n \rightarrow \infty} \int_{\mathbb{R}^{d}} \exp \left(-\frac{i \epsilon\left(p_{n-1}^{2} / 2 m+V_{2}\left(p_{n-1}\right)\right)}{\hbar}\right) \cdot\left(\exp \left(-\frac{i \epsilon V_{1}}{\hbar}\right)\right. \\
& \left.\times\left(\exp \left(-\frac{i \epsilon\left(p^{2} / 2 m+V_{2}\right)}{\hbar}\right) \exp \left(-\frac{i \epsilon\left(V_{1}\right)}{\hbar}\right)\right)^{n-1} \psi_{0}\right)\left(p_{1}\right) \frac{\exp \left(i \frac{x p_{n-1}}{\hbar}\right)}{(2 \pi \hbar)^{d / 2}} \mathrm{~d} p_{n-1} \\
= & \lim _{n \rightarrow \infty} \int_{\mathbb{R}^{2 d}} \exp \left(-\frac{i \epsilon\left(p_{n-1}^{2} / 2 m+V_{2}\left(p_{n-1}\right)\right)}{\hbar}\right) \exp \left(-\frac{i \epsilon V_{1}\left(x_{n-1}\right.}{\hbar}\right) \\
& \cdot\left(\left(\exp \left(-\frac{i \epsilon\left(p^{2} / 2 m+V_{2}\right)}{\hbar}\right) \exp \left(-\frac{i \epsilon\left(V_{1}\right)}{\hbar}\right)\right)^{n-1} \psi_{0}\right)\left(x_{n-1}\right) \frac{\exp \left(i \frac{x p_{n-1}}{\hbar}\right)}{(2 \pi \hbar)^{d / 2}} \\
& \times \frac{\exp \left(-i \frac{x_{n-1} p_{n-1}}{\hbar}\right)}{(2 \pi \hbar)^{d / 2}} \mathrm{~d} p_{n-1} \mathrm{~d} x_{n-1} \\
= & \lim _{n \rightarrow \infty}\left(\frac{1}{\sqrt{2 \pi \hbar}}\right)^{2 n d} \cdot \int_{\mathbb{R}^{2 n d}} \exp \left(-\frac{i \epsilon}{\hbar} \sum_{j=0}^{n-1}\left(\frac{p_{j}^{2}}{2 m}+V_{1}\left(x_{j}\right)+V_{2}\left(p_{j}\right)\right.\right. \\
& \left.-p_{j} \frac{\left(x_{j+1}-x_{j}\right)}{\epsilon}\right) \psi_{0}\left(x_{0}\right) \prod_{j=0}^{n-1} \mathrm{~d} p_{j} \mathrm{~d} x_{j},
\end{aligned}
$$

where $x_{n} \equiv x$.

Remark: The integrals above are to be understood as limits as $\Lambda \uparrow \mathbb{R}^{d}, n \rightarrow \infty$ in the $L^{2}\left(\mathbb{R}^{2 n d}\right)$ sense of the corresponding integrals over $\Lambda^{2 n d}$, with $\Lambda$ bounded (see Ref. 11). Formula (5) holds first as a strong $L^{2}$-limit, but then (possibly by subsequences) also for Lebesgue a.e.x. It also follows from this that (5) gives the solution to the Cauchy problem (1).

The latter expression suggests the following formula for the limit:

$$
\begin{gathered}
\psi(t, x)=\text { const } \int_{q(t)=x} e^{(i / \hbar) S(q, p)} \psi(0, q(0)) \mathrm{d} q \mathrm{~d} p, \\
S(q, p)=\int_{0}^{t} p(s) \dot{q}(s)-H(q(s), p(s)) \mathrm{d} s
\end{gathered}
$$

which does not yet have a mathematical meaning. It will be rigorously defined in Secs. III and IV. 


\section{OSCILLATORY INTEGRALS AND THE CAMERON MARTIN FORMULA}

In this section we recall for later use some known results, for more details we refer to Refs. 2, 3 , and 8 .

\section{A. Finite dimensional oscillatory integrals}

Let us consider the finite dimensional real Hilbert space $\mathbb{R}^{n}$, whose elements are denoted by $x, y \in \mathbb{R}^{n}$ and the scalar product with $\langle x, y\rangle$. Let $T: \mathbb{R}^{n} \rightarrow \mathbb{R}^{n}$ be a nondegenerate symmetric operator.

Definition 1: A function $f: \mathbb{R}^{n} \rightarrow \mathrm{C}$ is Fresnel integrable with respect to $T$ if and only if for each $\phi \in \mathcal{S}\left(\mathbb{R}^{n}\right)$ such that $\phi(0)=1$ the limit

$$
\lim _{\epsilon \rightarrow 0} \int e^{i\langle x, T x\rangle} f(x) \phi(\epsilon x) \mathrm{d} x
$$

exists and is independent of $\phi$. In this case the limit is called the Fresnel integral of $f$ with respect to $T$ and denoted by

$$
\int e^{i\langle x, T x\rangle} f(x) \mathrm{d} x
$$

There is an important class $\mathcal{F}\left(\mathbb{R}^{n}\right)$ of Fresnel integrable functions: those which are Fourier transforms of complex bounded variation measures on $\mathbb{R}^{n}, \mathcal{M}\left(\mathbb{R}^{n}\right)$ :

$$
f \in \mathcal{F}\left(\mathbb{R}^{n}\right) \Leftrightarrow f(x)=\int e^{i\langle x, \alpha\rangle} \mu_{f}(d \alpha), \quad \mu_{f} \in \mathcal{M}\left(\mathbb{R}^{n}\right), \quad x \in \mathbb{R}^{n} .
$$

$\mathcal{F}\left(\mathbb{R}^{n}\right)$ contains in particular $\mathcal{S}\left(\mathbb{R}^{n}\right)$, hence it is also dense in $L^{2}\left(\mathbb{R}^{n}\right)$. In this case the Parseval equality gives us the following expression for the limit (7):

$$
(2 \pi i)^{-n / 2} \int e^{(i / 2)\langle x, T x\rangle} f(x) \mathrm{d} x=(\operatorname{det} T)^{-1 / 2} \int e^{(-i / 2)\left\langle x, T^{-1} x\right\rangle} \mu_{f}(\mathrm{~d} x) .
$$

Analogously one can define the normalized Fresnel integral by means of the following expression:

$$
\iint e^{(i / 2)\langle x, T x\rangle} f(x) \mathrm{d} x:=(\operatorname{det} T)^{1 / 2}(2 \pi)^{-n / 2} \int e^{(i / 2)\langle x, T x\rangle} f(x) \mathrm{d} x=\int e^{(-i / 2)\left\langle x, T^{-1} x\right\rangle} \mu_{f}(\mathrm{~d} x)
$$

Note that if we substitute into the latter definition the function $f=1$ we have $\int e^{(i / 2)\langle x, T x\rangle} f(x) \mathrm{d} x$ $=1$.

\section{B. Infinite dimensional oscillatory integrals}

Let us consider an infinite dimensional real Hilbert space of paths $\mathcal{H}$ whose elements are denoted by $\gamma, \eta \in \mathcal{H}$ and the scalar product by $\langle\gamma, \eta\rangle$. Let $P_{n}$ be a sequence of projectors on $n$-dimensional subspaces of $\mathcal{H}$ such that $P_{n} \leqslant P_{n+1}$ [i.e., $P_{n+1}=I$ on $\left.P_{n}(\mathcal{H})\right]$ and $P_{n} \rightarrow I$ strongly as $n \rightarrow \infty$; let $f: \mathcal{H} \rightarrow \mathrm{C}$ be a function on $\mathcal{H}$ and let $T: D(T) \subseteq \mathcal{H} \rightarrow \mathcal{H}$ be a self-adjoint invertible operator.

Definition 2: A function $f: \mathcal{H} \rightarrow \mathrm{C}$ is Fresnel integrable with respect to $T$ if and only if for each $n$ the following finite dimensional integral

$$
\int_{P_{n} \mathcal{H}} e^{i\left\langle P_{n} \gamma, T P_{n} \gamma\right\rangle} f\left(P_{n} \gamma\right) \mathrm{d} P_{n} \gamma
$$

is well defined and the limit 


$$
\lim _{n \rightarrow \infty} \int_{P_{n} \mathcal{H}} e^{i\left\langle P_{n} \gamma, T P_{n} \gamma\right\rangle} f\left(P_{n} \gamma\right) \mathrm{d} P_{n} \gamma
$$

exists and is independent of the sequence $\left\{P_{n}\right\}$.

In this case the limit is called the Fresnel integral of $f$ with respect to $T$ and is denoted by

$$
\int e^{i\langle\gamma, T \gamma\rangle} f(\gamma) \mathrm{d} \gamma
$$

Equation (8) is called the normalized finite dimensional approximation of this Fresnel integral.

One can prove that if $f \in \mathcal{F}(\mathcal{H})$ then $f \circ P_{n} \in \mathcal{F}\left(P_{n}(\mathcal{H})\right)$. Moreover $f$ is Fresnel integrable and the Cameron-Martin-type formula holds:

$$
\int e^{(i / 2)\langle\gamma, T \gamma\rangle} f(\gamma) \mathrm{d} \gamma=\int_{\mathcal{H}} e^{-(i / 2)\left\langle\gamma, T^{-1} \gamma\right\rangle} \mu_{f}(\mathrm{~d} \gamma),
$$

see Refs. 8 and 3.

\section{PHASE SPACE FEYNMAN FUNCTIONAL}

Let us consider again the expression (6) in the particular case of the free particle, namely when the Hamiltonian is just the kinetic energy: $H=p^{2} / 2 m$. In this case we have heuristically

$$
\psi(t, x)=\text { const } \int_{q(t)=x} \exp \left(\frac{i}{\hbar} \int_{0}^{t}\left(p(s) \dot{q}(s)-p(s)^{2} / 2 m\right) \mathrm{d} s\right) \psi(0, q(0)) \mathrm{d} q \mathrm{~d} p .
$$

We can give a precise meaning to this expression: under suitable hypothesis on the initial wave function $\psi_{0}$, it is an infinite dimensional oscillatory integral. ${ }^{14}$ In fact, following Ref. 15, let us introduce the Hilbert space $\mathcal{H}_{t} \times \mathcal{L}_{t}$, namely the space of paths in the $d$-dimensional phase space $(q(s), p(s))_{s \in[0, t]}$, such that the path $(q(s))_{s \in[0, t]}$ belongs to the Cameron-Martin space $\mathcal{H}_{t}$, namely to the space of the absolutely continuous functions $q$ from $[0, t]$ to $\mathbb{R}^{d}$ such that $q(t)$ $=0$ and $\dot{q} \in \mathcal{L}_{2}\left([0, t], \mathbb{R}^{d}\right)$, with inner product $\left\langle q_{1}, q_{2}\right\rangle=\int_{0}^{t} \dot{q}_{1}(s) \dot{q}_{2}(s) \mathrm{d} s$, while the path in the momentum space $(p(s))_{s \in[0, t]}$ belongs to $\mathcal{L}_{t}=\mathcal{L}_{2}\left([0, t], \mathbb{R}^{d}\right)$. $\mathcal{H}_{t} \times \mathcal{L}_{t}$ is an Hilbert space with the natural inner product

$$
\langle q, p ; Q, P\rangle=\int_{0}^{t} \dot{q}(s) \dot{Q}(s) \mathrm{d} s+\int_{0}^{t} p(s) P(s) \mathrm{d} s .
$$

Let us introduce the following bilinear form:

$$
[q, p ; Q, P]=\int_{0}^{t} \dot{q}(s) P(s) \mathrm{d} s+\int_{0}^{t} p(s) \dot{Q}(s) \mathrm{d} s-\int_{0}^{t} p(s) P(s) \mathrm{d} s=\langle q, p ; A(Q, P)\rangle,
$$

where $A$ is the following operator in $\mathcal{H}_{t} \times \mathcal{L}_{t}$ :

$$
A(Q, P)(s)=\left(\int_{t}^{s} P(u) \mathrm{d} u, \dot{Q}(s)-P(s)\right) .
$$

$A(Q, P)$ is densely defined, e.g., on $C^{1}\left([0, t] ; \mathbb{R}^{d}\right) \times C^{1}\left([0, t] ; \mathbb{R}^{d}\right)$. Moreover $A(Q, P)$ is invertible with inverse given by

$$
A^{-1}(Q, P)(s)=\left(\int_{t}^{s} P(u) \mathrm{d} u+Q(s), \dot{Q}(s)\right)
$$


(on the range of $A$ ).

Now expression (6) can be realized rigorously as

$$
\int_{\mathcal{H}_{t} \times \mathcal{L}_{t}} e^{(i / 2 \hbar)\langle q, p ; A(q, p)\rangle} \psi(0, q(0)+x) \mathrm{d} q \mathrm{~d} p,
$$

where $q+x$ denotes the traslated path $q(s) \rightarrow q(s)+x$, and the normalized integral is defined by (10).

In this case the heuristic expression (6) is well defined through Lie-Trotter product formula, namely as the limit of a sequence of finite dimensional integrals, as we saw in Sec. II. We are now going to show that it is also the limit of a sequence of finite dimensional oscillatory integrals in the sense of definition 2.

Let us consider a sequence of partitions $\pi_{n}$ of the interval $[0, t]$ into $n$ subintervals of amplitude $\epsilon \equiv t / n$ :

$$
t_{0}=0, t_{1}=\epsilon, \ldots, t_{i}=i \epsilon, \ldots, t_{n}=n \epsilon=t .
$$

To each $\pi_{n}$ we associate a projector $P_{n}: \mathcal{H}_{t} \times L_{t} \rightarrow: \mathcal{H}_{t} \times L_{t}$ onto a finite dimensional subspace of $\mathcal{H}_{t} \times L_{t}$, namely the subspace of polygonal paths. In other words each projector $P_{n}$ acts on a phase space path $(q, p) \in \mathcal{H}_{t} \times \mathcal{L}_{t}$ in the following way:

$$
P_{n}(q, p)(s)=\left(\sum_{i=1}^{n} \chi_{\left[t_{i-1}, t_{i}\right]}(s)\left(q\left(t_{i-1}\right)+\frac{\left(q\left(t_{i}\right)-q\left(t_{i-1}\right)\right.}{t_{i}-t_{i-1}}\left(s-t_{i-1}\right)\right), \sum_{i=1}^{n} \chi_{\left[t_{i-1}, t_{i}\right]}(s) p_{i}\right),
$$

where

$$
p_{i}=\frac{\int_{t_{i-1}}^{t_{i}} p(s) \mathrm{d} s}{t_{i}-t_{i-1}}=\frac{1}{\epsilon} \int_{t_{i-1}}^{t_{i}} p(s) \mathrm{d} s .
$$

Theorem 1: For each $n \in \mathbb{N}, P_{n}$ is a projector in $\mathcal{H}_{t} \times \mathcal{L}_{t}$. Moreover for $n \rightarrow \infty P_{n} \rightarrow I$ as a bounded operator.

Proof: $P_{n}$ is symmetric, indeed for all $(Q, P) \in \mathcal{H}_{t} \times \mathcal{L}_{t}$ and all $(q, p) \in \mathcal{H}_{t} \times \mathcal{L}_{t}$,

$$
\begin{aligned}
\left\langle Q, P ; P_{n}(q, p)\right\rangle & =\int_{0}^{t} \dot{Q}(s) \sum_{i=1}^{n} \chi_{\left[t_{i-1}, t_{i}\right]}(s) \frac{\left(q\left(t_{i}\right)-q\left(t_{i-1}\right)\right.}{t_{i}-t_{i-1}} \mathrm{~d} s+\int_{0}^{t} P(s) \sum_{i=1}^{n} \chi_{\left[t_{i-1}, t_{i}\right]}(s) p_{i} \mathrm{~d} s \\
& =\sum_{i=1}^{n} \frac{\left(q\left(t_{i}\right)-q\left(t_{i-1}\right)\left(Q\left(t_{i}\right)-Q\left(t_{i-1}\right)\right.\right.}{t_{i}-t_{i-1}}+\sum_{i=1}^{n} \frac{\int_{t_{i-1}}^{t_{i}} p(s) \mathrm{d} s \int_{t_{i-1}}^{t_{i}} P(s) \mathrm{d} s}{t_{i}-t_{i-1}} \\
& =\left\langle P_{n}(Q, P) ; q, p\right\rangle .
\end{aligned}
$$

$P_{n}^{2}=P_{n}$, indeed

$$
\begin{aligned}
P_{n}^{2}(q, p)(s) & =\left(\sum_{i=1}^{n} \chi_{\left[t_{i-1}, t_{i}\right]}(s)\left(q\left(t_{i-1}\right)+\frac{\left(q\left(t_{i}\right)-q\left(t_{i-1}\right)\right.}{t_{i}-t_{i-1}}\left(s-t_{i-1}\right)\right), \sum_{i=1}^{n} \chi_{\left[t_{i-1}, t_{i}\right]}(s) p_{i}\right) \\
& =P_{n}(q, p)(s)
\end{aligned}
$$

$\forall(q, p) \in \mathcal{H}_{t} \times \mathcal{L}_{t},\left\|P_{n}(q, p)-(q, p)\right\| \rightarrow 0$ as $n \rightarrow \infty:$

Let us consider the subset $\mathcal{K} \subseteq \mathcal{H}_{t} \times \mathcal{L}_{t}, \mathcal{K}=\left\{(q, p) \in \mathcal{H}_{t} \times \mathcal{L}_{t}:\left\|P_{n}(q, p)-(q, p)\right\| \rightarrow 0, n \rightarrow \infty\right\}$. It is enough to prove that the closure of $\mathcal{K}$ is $\mathcal{H}_{t} \times \mathcal{L}_{t}$. To prove this it is sufficient to show that $\mathcal{K}$ 
is a closed subspace of $\mathcal{H}_{t} \times \mathcal{L}_{t}$ and contains a dense subset of $\mathcal{H}_{t} \times \mathcal{L}_{t}$. This follows from the density of the piecewise linear paths in $\mathcal{H}_{t}$ and the density of the piecewise constant paths in $\mathcal{L}_{t}$ (see, e.g., Ref. 14).

Theorem 2: Let the function $(q, p) \rightarrow \psi_{0}(x+q(0)), \psi_{0} \in \mathcal{S}\left(\mathbb{R}^{d}\right)$, be Fresnel integrable with respect to $A$ [with A defined by (12)]. Then the phase space Feynman path integral, namely the limit

$$
\lim _{n \rightarrow \infty} \int_{P_{n}\left(\mathcal{H}_{t} \times \mathcal{L}_{t}\right)} e^{(i / 2 \hbar)\left\langle P_{n}(q, p), A P_{n}(q, p)\right\rangle} \psi_{0}(x+q(0)) \mathrm{d} P_{n}(q, p)
$$

coincides with the limit (5), namely with the solution of the Schrödinger equation with a free Hamiltonian.

Proof: The result follows by direct computation, indeed:

$$
\begin{aligned}
& \int_{P_{n}\left(\mathcal{H}_{t} \times \mathcal{L}_{t}\right)} e^{(i / 2 \hbar)\left\langle P_{n}(q, p), A P_{n}(q, p)\right\rangle} \psi_{0}(x+q(0)) \mathrm{d} P_{n}(q, p) \\
& =\left(\frac{1}{\sqrt{2 \pi \hbar}}\right)^{2 n d} \int_{\mathbb{R}^{2 n d}} \exp \left(-\frac{i \epsilon}{\hbar} \sum_{j=0}^{n-1}\left(\frac{p_{j}^{2}}{2 m}-p_{j} \frac{\left(x_{j+1}-x_{j}\right)}{\epsilon}\right)\right) \psi_{0}\left(x_{0}\right) \prod_{j=0}^{n-1} \mathrm{~d} p_{j} \mathrm{~d} x_{j},
\end{aligned}
$$

and the two limits (5) and (14) coincide. Indeed (14) is a pointwise limit by hypothesis. On the other hand (5) is a limit in $L_{2}$ sense, hence, passing if necessary to a subsequence, it is also a pointwise limit.

Remark 1: The latter result is equivalent to the "traditional" formulation of the Feynman path integral in the configuration space. Indeed it can be obtained by means of Fubini theorem ${ }^{2}$ and an integration with respect to the momentum variables:

$$
\begin{aligned}
& \lim _{n \rightarrow \infty}\left(\frac{1}{\sqrt{2 \pi \hbar}}\right)^{2 n d} \int_{\mathbb{R}^{2 n d}} \exp \left(-\frac{i \epsilon}{\hbar} \sum_{j=0}^{n-1}\left(\frac{p_{j}^{2}}{2 m}-p_{j} \frac{\left(x_{j+1}-x_{j}\right)}{\epsilon}\right)\right) \psi_{0}\left(x_{0}\right) \prod_{j=0}^{n-1} \mathrm{~d} p_{j} \mathrm{~d} x_{j} \\
& =\lim _{n \rightarrow \infty}\left(\frac{1}{\sqrt{2 \pi i \hbar}}\right)^{n d} \int_{\mathbb{R}^{n d}} \exp \left(-\frac{i \epsilon}{\hbar} \sum_{j=0}^{n-1} m \frac{\left(x_{j+1}-x_{j}\right)^{2}}{2 \epsilon^{2}}\right) \psi_{0}\left(x_{0}\right) \prod_{j=0}^{n-1} \mathrm{~d} x_{j} .
\end{aligned}
$$

The latter expression yields the Feynman functional on the configuration space, i.e., heuristically const $\int \exp \left(\int_{0}^{t} \mathcal{L}(q(s), \dot{q}(s)) \mathrm{d} s\right) \mathrm{d} q(\mathcal{L}$ being the classical Lagrangian density).

Remark 2: The integration with respect to the momentum variables might seem to be superfluous, but it is very useful when we introduce a potential depending on the momentum.

Theorem 3: Let us consider a semibounded potential $V$ depending explicitly on the momentum: $V=V(p)$ and the corresponding quantum mechanical Hamiltonian $H=-\left(\hbar^{2} / 2\right) \Delta+V(p)$. Let us suppose $H$ is an essentially self-adjoint operator on $\mathcal{L}_{2}\left(\mathbb{R}^{d}\right)$. Let the function $(q, p)$ $\rightarrow \exp \left(-(i / \hbar) \int_{0}^{t} V\left(P_{n}(p(s))\right) \mathrm{d} s\right) \psi_{0}(x+q(0))$ be Fresnel integrable with respect to the operator $A$, with A defined by (12). Then the solution to the Schrödinger equation

$$
\left\{\begin{array}{l}
\dot{\psi}=-\frac{i}{\hbar} H \psi, \\
\psi(0, x)=\psi_{0}(x), \quad \psi_{0} \in \mathcal{S}\left(\mathrm{R}^{d}\right)
\end{array}\right.
$$

is given by the phase space path integral 


$$
\begin{aligned}
& \lim _{n \rightarrow \infty} \int_{P_{n}\left(\mathcal{H}_{t} \times \mathcal{L}_{t}\right)} \exp \left(\frac{i}{2 \hbar}\left\langle P_{n}(q, p), A P_{n}(q, p)\right\rangle\right) \exp \left(-\frac{i}{\hbar} \int_{0}^{t} V\left(P_{n}(p(s))\right) \mathrm{d} s\right) \\
& \quad \times \psi_{0}(x+q(0)) \mathrm{d} P_{n}(q, p) .
\end{aligned}
$$

Proof: We can proceed in a completely analogous way as in the proof of Theorem 2, therefore we shall omit the details.

\section{THE PHASE SPACE FEYNMAN-KAC FORMULA}

Let us consider a classical potential $V$ depending both on the position $Q \in \mathbb{R}^{d}$ and on the momentum $P \in \mathbb{R}^{d}$, but of the special form: $V=V(Q, P)=V_{1}(Q)+V_{2}(P)$ (The general case presents problems due to the noncommutativity of the quantized expression of $Q$ and $P$ ), for a different approach with more general Hamiltonians see Ref. 16. Moreover let us suppose the function $f: \mathcal{H}_{t} \times \mathcal{L}_{t} \rightarrow \mathrm{C}$,

$$
f(q, p)=\psi_{0}(x+q(0)) \exp \left(-\frac{i}{\hbar} \int_{0}^{t} V(q(s)+x, p(s)) \mathrm{d} s\right), \quad \psi_{0} \in \mathcal{S}\left(\mathbb{R}^{d}\right)
$$

is the Fourier transform of a complex bounded variation measure $\mu_{f}$ on $\mathcal{H}_{t} \times \mathcal{L}_{t}:{ }^{17}$

$$
f(q, p)=\int_{\mathcal{H}_{t} \times \mathcal{L}_{t}} e^{i\langle q, p ; Q, P\rangle} \mathrm{d} \mu_{f}(Q, P) .
$$

Under additional assumptions on $V_{1}$ and $V_{2}$ we shall see that the phase space Feynman path integral of the function $f$ can be computed and is given by

$$
\begin{aligned}
& \int_{\mathcal{H}_{t} \times \mathcal{L}_{t}} \exp \left(\frac{i}{2 \hbar}\langle q, p ; A(q, p)\rangle\right) \exp \left(-\frac{i}{\hbar} \int_{0}^{t} V(q(s)+x, p(s)) \mathrm{d} s\right) \psi(0, q(0)+x) \mathrm{d} q \mathrm{~d} p \\
& =\int_{\mathcal{H}_{t} \times \mathcal{L}_{t}} \exp \left(\frac{-i \hbar}{2}\left\langle q, p ; A^{-1}(q, p)\right\rangle\right) \mathrm{d} \mu_{f}(q, p) .
\end{aligned}
$$

This follows from Sec. III together with the following.

Lemma 2: Let us consider a potential $V(Q, P)=V_{1}(Q)+V_{2}(P)$ and an initial wave function $\psi_{0}$ such that $V_{1}, \quad \psi_{0} \in \mathcal{F}\left(\mathbb{R}^{d}\right)$ and the function $p(s)_{s \in[0, t]} \rightarrow \int_{0}^{t} V_{2}(p(s)) \mathrm{d} s \in \mathcal{F}\left(\mathcal{L}_{t}\right)$. Then the functional

$$
f(q, p)=\psi_{0}(x+q(0)) \exp \left(-\frac{i}{\hbar} \int_{0}^{t} V(q(s)+x, p(s)) \mathrm{d} s\right)
$$

belongs to $\mathcal{F}\left(\mathcal{H}_{t} \times \mathcal{L}_{t}\right)$.

Proof: $f(q, p)$ is the product of two functions: the first, say $f_{1}$, depends only on the first variable $q$, while the second $f_{2}$ depends only on the variable $p$, more precisely

$$
f_{1}(q)=\psi_{0}(x+q(0)) \exp \left(-\frac{i}{\hbar} \int_{0}^{t} V_{1}(q(s)+x) \mathrm{d} s\right), \quad f_{2}(p)=\exp \left(-\frac{i}{\hbar} \int_{0}^{t} V_{2}(p(s)) \mathrm{d} s\right) .
$$

Under the given hypothesis on $V_{1}$ and $\psi_{0}, f_{1}$ belongs to $\mathcal{F}\left(\mathcal{H}_{t}\right)$. The proof is given for instance in Ref. 2. For $f_{2}$ one must pay more attention: indeed the same proof given for $f_{1}$ does not work, as $f_{2}$ is defined on a different Hilbert space and we have to require explicitly that $\int_{0}^{t} V_{2}(p(s)) \mathrm{d} s \in \mathcal{F}\left(\mathcal{L}_{t}\right)$. Under this hypothesis one can easily prove that (see Ref. 2) $f_{2} \in \mathcal{F}\left(\mathcal{L}_{t}\right)$. 
Now if $f_{1}=\hat{\mu}_{f_{1}} \in \mathcal{F}\left(\mathcal{H}_{t}\right), f_{1}$ can be extended to a function, denoted again by $f_{1}$, in $\mathcal{F}\left(\mathcal{H}_{t}\right.$ $\left.\times \mathcal{L}_{t}\right)$ : it is the Fourier transform of the product measure on $\mathcal{H}_{t} \times \mathcal{L}_{t}$ of $\mu_{f_{1}}(\mathrm{~d} q)$ and $\delta_{0}(\mathrm{~d} p)$. The same holds for $f_{2}=\hat{\mu}_{f_{2}}: f_{2}=\left(\delta_{0}(\mathrm{~d} q) \mu_{f_{2}}(\mathrm{~d} p)\right)$.

Finally, as $\mathcal{F}\left(\mathcal{H}_{t} \times \mathcal{L}_{t}\right)$ is a Banach algebra, ${ }^{2}$ the product of two elements $f_{1} f_{2}$ is again an element of $\mathcal{F}\left(\mathcal{H}_{t} \times \mathcal{L}_{t}\right)$ : more precisely it is the Fourier transform of the convolution of the two measures in $\mathcal{M}\left(\mathcal{H}_{t} \times \mathcal{L}_{t}\right)$ corresponding to $f_{1}$ and $f_{2}$, respectively., and the conclusion follows. The next theorem shows that the above oscillatory integral (16) gives the solution to the Schrödinger equation.

Theorem 4: Let us consider the following Hamiltonian

$$
H(Q ; P)=\frac{P^{2}}{2}+V_{1}(Q)+V_{2}(P)
$$

in $L^{2}\left(R^{d}\right)$ and the corresponding Schrödinger equation

$$
\left\{\begin{array}{l}
\dot{\psi}=-\frac{i}{\hbar} H \psi, \\
\psi(0, x)=\psi_{0}(x), \quad x \in \mathbb{R}^{d} .
\end{array}\right.
$$

Let us suppose that $V_{1}, \psi_{0} \in \mathcal{F}\left(\mathbb{R}^{d}\right)$ and $\int_{0}^{t} V_{2}(p(s)) \mathrm{d} s \in \mathcal{F}\left(\mathcal{L}_{t}\right)$. Then the solution to the Cauchy problem (17) is given by the phase space Feynman path integral:

$$
\int_{\mathcal{H}_{t} \times \mathcal{L}_{t}} \exp \left(\frac{i}{2 \hbar}\langle q, p ; A(q, p)\rangle\right) \exp \left(-\frac{i}{\hbar} \int_{0}^{t}\left(V_{1}(q(s)+x)+V_{2}(p(s))\right) \mathrm{d} s\right) \psi(0, q(0)+x) \mathrm{d} q \mathrm{~d} p .
$$

Proof: We follow the proof given by Elworthy and Truman in Ref. 3 .

For $0 \leqslant u \leqslant t$ let $\mu_{u}\left(V_{1}, x\right) \equiv \mu_{u}, \nu_{u}^{t}\left(V_{1}, x\right) \equiv \nu_{u}^{t}, \eta_{u}^{t}\left(V_{2}\right) \equiv \eta_{u}^{t}$, and $\mu_{0}(\psi)$ be the measures on $\mathcal{H}_{t} \times \mathcal{L}_{t}$ whose Fourier transforms when evaluated at $(q, p) \in \mathcal{H}_{t} \times \mathcal{L}_{t}$ are

$$
V_{1}(x+q(u)), \quad \exp \left(-i \int_{u}^{t} V_{1}(x+q(s)) \mathrm{d} s\right), \quad \exp \left(-i \int_{u}^{t} V_{2}(p(s)) \mathrm{d} s\right), \quad \psi_{0}(q(0)+x) .
$$

We set

$$
\begin{aligned}
U(t) \psi_{0}(x)= & \int_{\mathcal{H}_{t} \times \mathcal{L}_{t}} \exp \left(\frac{i}{2 \hbar}\langle q, p ; A(q, p)\rangle\right) \\
& \times \exp \left(-\frac{i}{\hbar} \int_{0}^{t}\left(V_{1}(q(s)+x)+V_{2}(p(s))\right) \mathrm{d} s\right) \psi(0, q(0)+x) \mathrm{d} q \mathrm{~d} p
\end{aligned}
$$

and

$$
\begin{aligned}
U_{0}(t) \psi_{0}(x)= & \int_{\mathcal{H}_{t} \times \mathcal{L}_{t}} \exp \left(\frac{i}{2 \hbar}\langle q, p ; A(q, p)\rangle\right) \\
& \times \exp \left(-\frac{i}{\hbar} \int_{0}^{t} V_{2}(p(s))\right) \mathrm{d} s \psi(0, q(0)+x) \mathrm{d} q \mathrm{~d} p .
\end{aligned}
$$

By Sec. III we have

$$
U(t) \psi_{0}(x)=\int_{\mathcal{H}_{t} \times \mathcal{L}_{t}} e^{(-i \hbar / 2)\left\langle q, p ; A^{-1}(q, p)\right\rangle}\left(\eta_{0}^{t *} \nu_{0}^{t *} \mu_{0}(\psi)\right)(\mathrm{d} q \mathrm{~d} p) .
$$

Now, if $\left\{\mu_{u}: a \leqslant u \leqslant t\right\}$ is a family in $\mathcal{M}\left(\mathcal{H}_{t} \times \mathcal{L}_{t}\right)$, we shall let $\int_{a}^{b} \mu_{u} \mathrm{~d} u$ denote the measure on $\mathcal{H}_{t} \times \mathcal{L}_{t}$ given by 


$$
f \rightarrow \int_{a}^{b} \int_{\mathcal{H}_{t} \times \mathcal{L}_{t}} f(q, p) \mathrm{d} \mu_{u}(q, p) \mathrm{d} u
$$

whenever it exists.

Since for any continuous path $q$,

$$
\exp \left(-i \int_{0}^{t} V_{1}(q(s)) \mathrm{d} s\right)=1-i \int_{0}^{t} V_{1}(q(u)) \exp \left(-i \int_{u}^{t} V_{1}(q(s)) \mathrm{d} s\right) \mathrm{d} u
$$

the following relation holds:

$$
\nu_{0}^{t}=\delta_{0}-i \int_{0}^{t}\left(\mu_{u}^{*} \nu_{u}^{t}\right) \mathrm{d} u
$$

where $\delta_{0}$ is the Dirac measure at $0 \in \mathcal{H}_{t}$.

Applying this relation to (18) we obtain

$$
\begin{aligned}
& U(t) \psi_{0}(x)=\int_{\mathcal{H}_{t} \times \mathcal{L}_{t}} \exp \left(\frac{-i \hbar}{2}\left\langle q, p ; A^{-1}(q, p)\right\rangle\right)\left(\eta_{0}^{t} * \mu_{0}(\psi)\right)(\mathrm{d} q \mathrm{~d} p) \\
& -i \int_{0}^{t} \int_{\mathcal{H}_{t} \times \mathcal{L}_{t}} \exp \left(\frac{-i \hbar}{2}\left\langle q, p ; A^{-1}(q, p)\right\rangle\right)\left(\eta_{0}^{t *} \mu_{u}\left(V_{1}, x\right) * \nu_{u}^{t} * \mu_{0}(\psi)\right)(\mathrm{d} q \mathrm{~d} p) \mathrm{d} u \\
& =U_{0}(t) \psi_{0}(x)-i \int_{0}^{t} \widetilde{\int} \mathcal{H}_{t} \times \mathcal{L}_{t} \exp \left(\frac{i}{2 \hbar}\langle q, p ; A(q, p)\rangle\right) \exp \left(-\frac{i}{\hbar} \int_{u}^{t} V_{1}(q(s)+x) \mathrm{d} s\right) \\
& \times \exp \left(-\frac{i}{\hbar} \int_{0}^{t} V_{2}(p(s))\right) \mathrm{d} s V_{1}(q(u)+x) \psi_{0}(q(0)+x) \mathrm{d} q \mathrm{~d} p \mathrm{~d} u .
\end{aligned}
$$

Now we have, by Fubini theorem for Fresnel integrals, ${ }^{2}$

$$
\begin{aligned}
\int \mathcal{H}_{t} \times \mathcal{L}_{t} \exp \left(\frac{i}{2 \hbar}\langle q, p ; A(q, p)\rangle\right) \exp \left(-\frac{i}{\hbar} \int_{u}^{t} V_{1}(q(s)+x) \mathrm{d} s\right) \exp \left(-\frac{i}{\hbar} \int_{0}^{t} V_{2}(p(s))\right) \mathrm{d} s . \\
V_{1}(q(u)+x) \psi_{0}(q(0)+x) \mathrm{d} q \mathrm{~d} p \\
=\int \mathcal{H}_{t-u} \times \mathcal{L}_{t-u} \exp \left(\frac{i}{2 \hbar}\langle q, p ; A(q, p)\rangle_{\mathcal{H}_{t-u} \times \mathcal{L}_{t-u}}\right) \\
\quad \times \exp \left(-\frac{i}{\hbar} \int_{0}^{t-u} V_{1}(q(s)+x) \mathrm{d} s\right)\left(\exp -\frac{i}{\hbar} \int_{0}^{t-u} V_{2}(p(s))\right) \\
\quad \times \operatorname{ds} V_{1}(q(0)+x) \int \mathcal{H}_{u} \times \mathcal{L}_{u} \exp \left(\frac{i}{2 \hbar}\left\langle q_{1}, p_{1} ; A\left(q_{1}, p_{1}\right)\right\rangle_{\left.\mathcal{H}_{u} \times \mathcal{L}_{u}\right)}\right) \\
\\
\quad \times \exp \left(-\frac{i}{\hbar} \int_{0}^{u} V_{2}\left(p_{1}(s)\right)\right) \mathrm{d} s \psi_{0}\left(q_{1}(0)\right) \mathrm{d} q_{1} \mathrm{~d} p_{1} \mathrm{~d} q \mathrm{~d} p p .
\end{aligned}
$$

Here $q \in \mathcal{H}_{t-u}$ and $q_{1} \in \mathcal{H}_{u}$ are the integration variables, and $\mathcal{H}_{s}$ denotes the Cameron-Martin space of paths $\gamma:[0, s] \rightarrow \mathbb{R}^{d}$.

We have 


$$
\begin{aligned}
U(t) \psi_{0}(x) & =U_{0}(t) \psi_{0}(x)-i \int_{0}^{t} U(t-u)\left(V_{1} U_{0}(u) \psi_{0}\right)(x) \mathrm{d} u \\
& =U_{0}(t) \psi_{0}(x)-i \int_{0}^{t} U(u)\left(V_{1} U_{0}(-u) U_{0}(t) \psi_{0}\right)(x) \mathrm{d} u
\end{aligned}
$$

The iterative solution of the latter integral equation is the convergent Dyson perturbation series for $U(t)$ with respect to $U_{0}(t)$, which proves the theorem.

Remark: We have stated our results for $\psi_{0} \in \mathcal{F}\left(\mathbb{R}^{d}\right)$. They can be extended by density to $L^{2}\left(\mathbb{R}^{d}\right)$.

\section{ACKNOWLEDGMENTS}

The authors thank Professor David Elworthy and Professor Luciano Tubaro for many interesting discussions and their very kind hospitality at the Mathematics Research Center, University of Warwick, and the Mathematics Institute, University of Trento. They also gratefully acknowledge the hospitality of the Institute of Applied Mathematics at the University of Bonn as well as financial support from SFB256(DFG).

${ }^{1}$ M. Reed and B. Simon, Methods of Modern Physics. Vol. 1, Functional Analysis (Academic, New York, 1975).

${ }^{2}$ S. Albeverio and R. J. Høegh-Krohn, Mathematical Theory of Feynman Path Integrals, Lecture Notes in Mathematics (Springer, Berlin, 1976).

${ }^{3}$ D. Elworthy and A. Truman, Ann. I.H.P. Phys. Theor. 41, 115 (1984).

${ }^{4}$ G. W. Johnson and M. L. Lapidus, The Feynman Integral and Feynman's Operational Calculus (Oxford University Press, New York, 2000).

${ }^{5}$ T. Hida, H. H. Kuo, J. Potthoff, and L. Streit, White Noise (Kluwer, Dordrecht, 1995).

${ }^{6}$ R. P. Feynman and A. R. Hibbs, Quantum Mechanics and Path Integrals (McGraw-Hill, New York, 1965).

${ }^{7}$ L. S. Schulman, Lecture on Path Integration World Scientific, River Edge, NJ, 1993).

${ }^{8}$ S. Albeverio and Z. Brzeźniak, J. Funct. Anal. 113, 177 (1993).

${ }^{9}$ V. P. Maslov and M. V. Fedoriuk, Semi-Classical Approximation in Quantum Mechanics (Reidel, Dordrecht, 1981).

${ }^{10}$ I. Daubechies and J. Klauder, J. Math. Phys. 28, 85 (1987).

${ }^{11}$ E. Nelson, J. Math. Phys. 5, 332-343 (1964).

${ }^{12}$ P. R. Chernoff, Product Formulas, Nonlinear Semigroups, and Addition of Unbounded Operators, Mem. Am. Math. Soc. 140 (1974).

${ }^{13}$ M. Reed, B. Simon, Methods of Modern Mathematical Physics, Vol. II, Fourier Analysis, Self-Adjointness (Academic, New York, 1975), Vol II.

${ }^{14}$ A. Truman, Springer Lecture Notes in Physics, edited by S. Albeverio et al. (Springer, New York, 1979), Vol. 106.

${ }^{15}$ A. Truman and T. Zastawniak, J. Korean Math. Soc. 38, 469 (2001).

${ }^{16}$ O. G. Smolyanov and E. T. Shavgulidze, Path Integrals (Moscow State University Press, Moscow, 1990) (in Russian).

${ }^{17}$ S. Albeverio and Z. Brzeźniak, J. Math. Phys. 36, 2135 (1995). 